\section{A reply to Robinson}

\section{WILLIAM R. UTTAL* \\ University of Western Australia, Nedlands, Western Australia 6009}

Robinson's criticisms appear to be based on misunderstandings of the methods and logic of my paper and those of other workers. They have been clarified point by point. It is concluded that there is still no direct evidence for the lateral inhibition hypothesis as an explanation of visual interactions.

(1) Much of the discussion Robinson (1971) presents is based upon a misunderstanding of the purpose and logic of my paper (Uttal, 1970). He says that I interpreted my results with dot patterns as "evidence against the notion that lateral inhibition is responsible for a broad range of short-term interference phenomena in vision." In fact, it was only on the basis of the fourth experiment that I discussed some of the things which have concerned me with regard to some of the current physiologizing about perceptual phenomena. I quote from my abstract: "The results indicate that none of the classic characteristics of lateral inhibitory interaction are obtained with this type of stimulus material. $A$ rather informal demonstration is then described that suggests further that lateral inhibitory interaction may also not be a satisfactory explanation of metacontrast and other kinds of masking that use continuous figures [italics added] [ Uttal, 1970, Abstract]."

In the latter part of my discussion, in which $I$ extend the idea emerging from Experiment 4, my argument dealt mainly with the weakness of the lateral inhibitory hypothesis in explaining nonoverlapping masking and not the concentric disk paradigm. In fact, it is there that I point out that the neurophysiological data and the results for overlapping stimuli do appear to correspond. To argue from displaced patterns to concentric disks may have been incorrect or not, but in any event $I$ argued neither from dots to metacontrast nor from dots and metacontrast to masking with concentric disks.

(2) Much of the latter half of Robinson's comments seem to be based on a misunderstanding of $\mathrm{my}$ method. I count five separate places in my paper where I allude to the fact that the mask of Experiment 1 was a special case and I also cross reference the difference in Experiment 2 to an earlier report. In Experiments 2 and 3 the masking dots were not omitted

*On leave from the University of Michigan during the academic year 1970-1971. Reprint requests should be addressed to $1042 \mathrm{MHRI}$, University of Michigan, Ann Arbor, Michigan 48103. from the central region in which the character appeared. His suggestion of an experiment in which a stimulus "is obscured by the insertion of dots in the target space, not around it" is exactly the condition of my Experiments 2 and 3 . His further suggestion to use spots of different sizes is potentially interesting but not germane.

(3) With regard to his interpretation of Békésy (1968), I believe it would be best to let Békésy speak for himself: ". . it can be demonstrated that stimuli with a small lateral spread do not produce large changes in the local sensation as a consequence of inhibition. If the stimuli consisted of two very sharp and narrow sections, even when the distance between the two-point stimuli is inside the width of the Mach band type of inhibition, the reduction in sensation magnitude is quite small. If we increase the distance between the two-point stimuli so that the Hering-type of inhibition is involved, then there is almost no inhibitory effect between the two-point stimuli present [ Békésy, 1968 , pp. 1495-1496]."

(4) With regard to the Fehrer (1966) paper, Robinson says: "[Uttal] did not cite Fehrer (1966), who not only used similar stimulus materials, obtained similar results, and concluded (as Uttal did) that masking increases with overlap and form similarity, but who, in addition, cited lateral inhibition as a possible mechanism for the effect."

(a) Fehrer's stimuli were continuously drawn alphabetic stimuli and her masks were either a pair of parallel lines or one or more outline boxes. Mine were dot patterns in all cases.

(b) Where Fehrer found substantial masking with her nonoverlapping stimuli, I found none in my Experiment 1 , the corresponding condition.

(c) Fehrer did not study overlap as an independent variable, nor did she make any claims that masking increases with overlap.

(d) With regard to her citation of lateral inhibitory interaction, her own words could not be clearer: "The recent impressive experiments dealing with spatial inhibitory influences on the activity of single retinal"or cortical cells (e.g., Hubel \& Wiesel, 1962; Ratliff, 1961) have employed stimulating conditions and time sequences so different from ours that any attempt to relate the findings to our data seems premature [Fehrer, 1966, p. 615]."

(5) Robinson makes two related statements with regard to my first experiment.

(a) "Neither metacontrast suppression nor backward masking occurs when stimuli are presented to disparate retinal loci separated by more than 2-3 deg"; and

(b) "In this setting, Uttal found that masking is essentially impossible when target and mask are separated by as great a spatial extent as he employed."

Even in Experiment 1, the dots of the character and the dots of the mask were separated by no more than $13 / 4 \mathrm{deg}$ (half the width of the character plus the $1 / 4$-in. dot-free zone) and for those characters with dots concentrated along the sides by only a few minutes of visual angle on the average. Therefore, our dots were well within the region of possible interaction even as defined by Robinson.

(6) There are a number of other minor points on which I believe Robinson to be incorrect. In one place, he comments on the significance of the differences between the three sets of data of Experiment 3. A reading of the caption of my Fig. 4 should convince the reader that the lack of significant difference to which I referred is between the monoptic ( $x$ ) and binocular $(\bullet)$ conditions, and that no reference was made to the significance of difference between these two sets of data (which were so close that only a single curve could be drawn for the two of them), on the one hand, and the third set of data-the dichoptic condition-on the other. I do not agree that a formal test of significance of the nondifference between the monoptic and binocular condition would have added anything in this instance.

In a related vein, what would have been gained by a statement of the "absolute detection rate"? The information is there to compute if anyone cares to. I had pointed out earlier that approximately 2,500 trials were pooled for each data point. A reader could calculate an absolute detection rate if he wished.

(7) In fairness to Robinson, he is correct that I did garble my description of the Schiller (1968) experiment. The sentence on p. 321 should have read "... are not always reflected by single cells." I came closer 
in the discussion to clarifying this point, but unfortunately did not succeed. His phrasing is the correct one. However, I believe my argument still holds. Once having demonstrated that the neural response and the psychophysical response can be dissociated in one context, other associations of this same neural activity with perceptual magnitudes become suspect.

In the few pages I have been allowed by the editors to reply, it is, of course, not possible to describe fully my reasons for believing the indirect arguments used by my colleagues to support the lateral interaction hypothesis are inadequate. Simply put, there is still no direct evidence. I have already presented a more complete discussion of my views elsewhere (Uttal, 1971), which the interested reader may find relevant.

\section{REFERENCES}

BÉKÉSY, G. v. Mach- and Hering-type lateral inhibition in vision. Vision Research, 1968, 8, 1483-1499.

FEHRER, E. Effect of stimulus similarity on retroactive masking. Journal of Experimental Psychology, 1966, 71, 612-615.
ROBINSON, D. N, Backward masking, disinhibition. and hypothesized neural networks. Perception \& Psychophysics, $1971,10,33-35$.

SCHILLER, P. H. Single unit analysis of backward visual masking and metacontrast in the cat lateral geniculate nucleus. Vision Research, 1968, 8, 855-866.

UTTAL, W. R. On the physiological basis of masking with dotted visual noise. Perception \& Psychophysics, 1970, 7, 321-327.

UTTAL, $\mathbf{W}, \mathfrak{R}$. The psychobiological silly season or what happens when neurophysiological data become psy chological theories. Journal of General Psychology, 1971, 84, 151-166.

(Accepted for publication January 21, 1971.) 